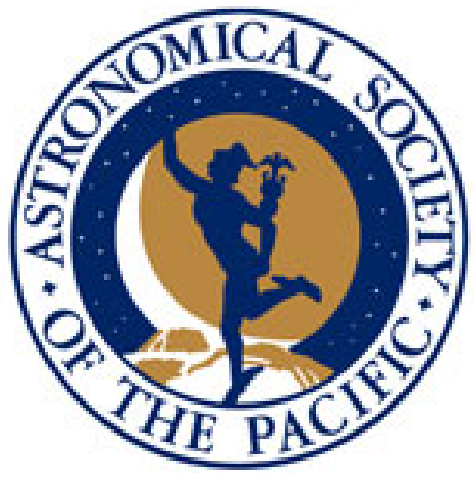

The Habitable Zone Gallery

Author(s): Stephen R. Kane and Dawn M. Gelino

Reviewed work(s):

Source: Publications of the Astronomical Society of the Pacific, Vol. 124, No. 914 (April 2012), pp. 323-328

Published by: The University of Chicago Press on behalf of the Astronomical Society of the Pacific

Stable URL: http://www.jstor.org/stable/10.1086/665271

Accessed: 11/05/2012 11:24

Your use of the JSTOR archive indicates your acceptance of the Terms \& Conditions of Use, available at http://www.jstor.org/page/info/about/policies/terms.jsp

JSTOR is a not-for-profit service that helps scholars, researchers, and students discover, use, and build upon a wide range of content in a trusted digital archive. We use information technology and tools to increase productivity and facilitate new forms of scholarship. For more information about JSTOR, please contact support@ jstor.org. 
Publications of the Astronomical Society of the Pacific, 124:323-328, 2012 April

(C) 2012. The Astronomical Society of the Pacific. All rights reserved. Printed in U.S.A.

\title{
The Habitable Zone Gallery
}

\author{
Stephen R. Kane and Dawn M. Gelino \\ NASA Exoplanet Science Institute, Caltech, MS 100-22, 770 South Wilson Avenue, Pasadena, CA 91125; skane@ipac.caltech.edu \\ Received 2012 January 17; accepted 2012 February 9; published 2012 March 2
}

\begin{abstract}
The Habitable Zone Gallery is a new service to the exoplanet community that provides habitablezone (HZ) information for each of the exoplanetary systems with known planetary orbital parameters. The service includes a sortable table with information on the percentage of orbital phase spent within the HZ, planetary effective temperatures, and other basic planetary properties. In addition to the table, we also plot the period and eccentricity of the planets with respect to their time spent in the HZ. The service includes a gallery of known systems that plot the orbits and the location of the HZ with respect to those orbits. Also provided are animations that aid in orbit visualization and provide the changing effective temperature for those planets in eccentric orbits. Here, we describe the science motivation, the underlying calculations, and the structure of the World Wide Web site.
\end{abstract}

\section{INTRODUCTION}

The field of exoplanets has undergone enormous diversification over the past 20 years. The reasons for this include (but are not limited to) new detection techniques, longer period baseline, smaller mass/radii sensitivity, and atmosphere detection and modeling. As a result of these developments, we are able to accurately characterize the orbits of exoplanets and to infer properties of their atmospheres' surface conditions. The sensitivity of radial velocity and transit surveys to planets at longer periods is fundamentally limited by the survey duration. Many of the radial velocity surveys are now pushing this detection threshold beyond orbital periods of 10 years and, with upgraded instruments, are sensitive to masses only a few times that of the Earth. This means that many of the known planets pass through or remain in the habitable zone (HZ) of their parent stars-some of these with potentially rocky surfaces or terrestrial-sized moons.

Keeping an accurate and exhaustive list of the known exoplanets is an increasingly difficult task, but there exist numerous electronic sources of information regarding exoplanets and their host stars. Notable examples are the Extrasolar Planets Encyclopaedia, ${ }^{1}$ the NASA Exoplanet Archive, ${ }^{2}$ and the Exoplanet Data Explorer (EDE). ${ }^{3}$ The EDE stores information only for those planets that have complete orbital solutions, typically from a Keplerian fit to radial velocity data acquired on those targets, and is thus very useful for this study.

The Habitable Zone Gallery ${ }^{4}$ (HZG) is a unique service that tracks the orbits of exoplanets in relation to the $\mathrm{HZ}$ of their host stars. This includes calculation of planetary temperatures,

\footnotetext{
${ }^{1}$ See http://exoplanet.eu/.

${ }^{2}$ See http://exoplanetarchive.ipac.caltech.edu/.

${ }^{3}$ http://exoplanets.org/.

${ }^{4}$ See http://www.hzgallery.org.
}

percentage of the orbital phase spent within the HZ, and figures/movies that depict planetary orbits with respect to the HZ. This allows for easy reference of interesting systems in this context and target selection for future investigations. This is also a useful tool for describing exoplanets in education and public outreach efforts. Here we detail the methodology used to perform the necessary calculations for generating the information on the HZG and the figures presented throughout.

\section{SCIENCE MOTIVATION}

The HZ is a key concept in our understanding of the conditions under which basic life can form and survive. On our own planet we find extreme conditions under which organisms are able to not only sustain metabolic processes, but also to thrive and grow. Thus, this understanding informs our precepts on how life formed in our own solar system and also the possibility of similar processes in exoplanetary systems. Although the concept of the $\mathrm{HZ}$ has been in the literature for some time, it is only within the past 20 years that complex atmospheric models have been developed to allow a rigorous examination of its nature. In particular, the response of different atmospheres to varying amounts of stellar flux allows the determination of HZ boundaries for known exoplanetary systems. The pioneering work on this was carried out by Kasting et al. (1993), with subsequent papers producing more analytical expressions for a variety of main-sequence stars (Jones \& Sleep 2010; Kasting et al. 1993; Selsis et al. 2007; Underwood et al.2003).

Exoplanet survey sensitivity is spreading in various directions: to lower masses, to longer periods, and to later and earlier spectral types. This broadening of the field opens up new areas to explore in terms of the habitable zones of these configurations. The topic of the HZ has become even more relevant in the wake of the results from the Kepler mission. Approaches 
to investigating the $\mathrm{HZ}$ of Kepler stars has been suggested by Kaltenegger \& Sasselov (2011), and many of the candidates released are within the HZ of their host stars (Borucki et al. 2011a, $2011 b$ ). The announcements of the Kepler-20 system (Fressin et al. 2012; Gautier et al. 2012) and Kepler-22b (Borucki et al. 2012) mark the crossing of a significant threshold in narrowing down the search for an Earth mass/radius planet that lies within the $\mathrm{HZ}$ of a solar-type star. Although these results inform us greatly as to the frequency of habitable terrestrial planets, the relative faintness of the Kepler sample makes more detailed follow-up difficult.

A new area of habitability being explored is that of terrestrial moons of giant exoplanets that lie within the $\mathrm{HZ}$ of their parent stars. This has been discussed in the context of the Kepler mission quite thoroughly (Kaltenegger 2010; Kipping et al. 2009). Based upon the statistics from our own solar system, it is natural to expect that many of the planets discovered via the radial velocity method do indeed harbor moons of various sizes and compositions. For the giant planets themselves, great progress is being made toward understanding the effective temperatures of these bodies, depending upon their albedos, thermal response, and atmospheric circulation (Kane \& Gelino 2010, 2011). Likewise for the moons in these systems, the habitability prospects will depend largely upon the conditions to which the primary planet is subjected.

The science motivation and use cases for the HZG can thus be summarized as follows:

1. To provide an interactive method by which users may visualize the orbits and habitable zones of known exoplanetary orbits.

2. To provide tools, graphics, and movies that can be easily imported into presentations to facilitate communication of these concepts in both public and scientific contexts.

3. To provide an interactive table tool that allows users to sort for planets that spend substantial amounts of time within the HZ and to thus aid in target selection for further studies.

4. Ultimately, and of particular interest to the authors, one would like to investigate the habitability of exoplanets and exomoons whose total energy budget varies with a cyclic nature, usually caused by orbital eccentricity and, consequently, variable stellar flux.

\section{HABITABLE ZONE AND ORBITS}

In order for the HZG to function, it requires the ability to calculate HZ parameters in a noninteractive fashion. Here, we describe the stellar and planetary information required and the subsequent calculations performed.

\subsection{Necessary Information}

The HZG uses a variety of stellar and planetary parameters as input to carry out the necessary calculations. The data for the planets listed in the HZG are primarily extracted from the
EDE, described in more detail by Wright et al. (2011). The full list of required parameters is shown in Table 1. As one might expect, not all required parameters are available in all cases. In order to provide as complete a list as possible, the HZG code attempts to fill in the data gaps using published material on known stellar and planetary properties. These are described in detail in $\S 3.2$.

\subsection{Assumptions and Missing Values}

The planetary temperature calculations are based upon parameters that are not available in all cases, and thus assumptions are occasionally made. The largest sources of uncertainties in the calculations are caused by the generally unknown radii of both the host star and the planet. In cases where the radius of the star is not available from other sources, we estimate the radius from the derived values of the surface gravity log $g$ using the relation

$$
\log g=\log \left(\frac{M_{\star}}{M_{\odot}}\right)-2 \log \left(\frac{R_{\star}}{R_{\odot}}\right)+\log g_{\odot},
$$

where $\log g_{\odot}=4.4374$ (Smalley 2005).

For nontransiting planets, a planetary radius measurement is invariably not available. The mass-radius relationship of exoplanets has been extensively studied, particularly with regard to composition models such as that described by Swift et al. (2012). To produce an estimate of planetary radii, we fit a simple model to the available data for confirmed transiting planets extracted using the EDE. The data are current as of 2012 January 3 and include 135 planets.

These data are plotted in Figure 1. For masses greater than $\sim 0.3$ Jupiter masses $\left(M_{\mathrm{J}}\right)$ the radii generally follow a linear model that approximates to $1 M_{\mathrm{J}}$. This is true for masses well into the brown dwarf regime (see, for example, the isochrone models of Baraffe et al. [2003]). However, there is a significant divergence from a linear model for masses less than $0.3 M_{\mathrm{J}}$. To account for this, we fit a power law to those data to obtain a

TABLE 1

REQUIRED INPUT PARAMETERS

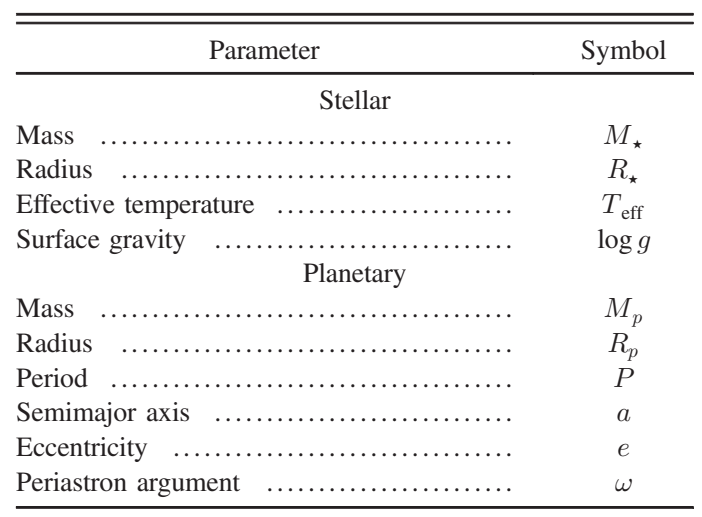




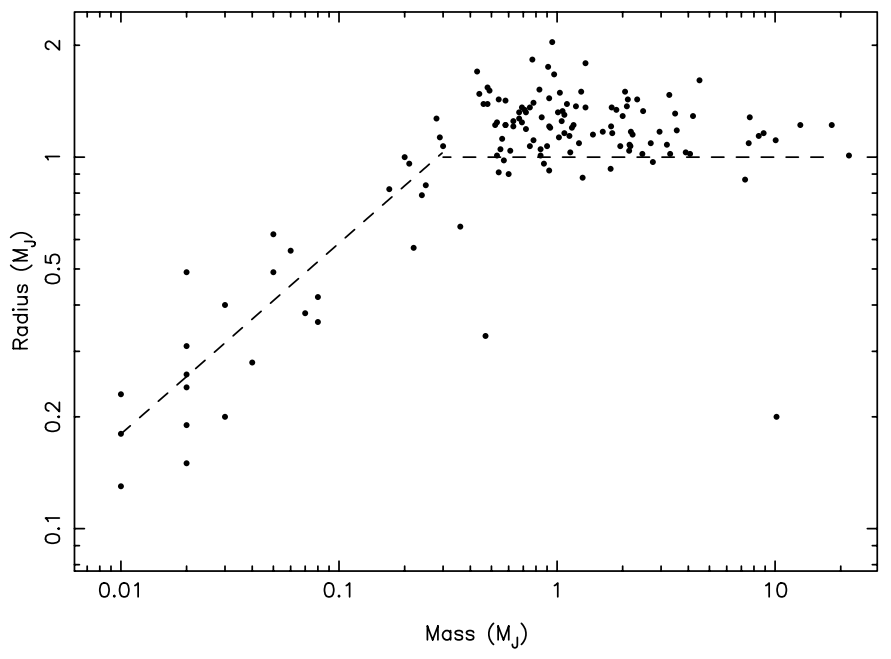

FIG. 1.-Mass and radius of known transiting planets. The dashed line indicates the model that the HZG uses to produce an approximation of the radius for nontransiting planets, where a power law is used for $M_{p}<0.3 M_{\mathrm{J}}$ and a constant value of $1.0 M_{\mathrm{J}}$ is implemented for $M_{p}>0.3$.

better first-order approximation of the radii of those objects. The overall model used is shown in Figure 1 as a dashed line. These radii may be used to calculate predicted planetary flux values at both infrared and optical wavelengths and also to estimate planetary densities for aid in further characterization scenarios. This assumes that the actual mass of the planets is close to the minimum mass deduced from the Keplerian orbital solutions. It is also worth noting that this sample is highly biased toward short-period orbits, typical of transiting planets, where the planetary radii can be inflated due to increased irradiation and tidal effects. Consequently, the median period of the plotted sample is relative low (3.41 days).

A crucial parameter for estimating the planetary effective temperatures is the planetary albedo: specifically, at infrared wavelengths. This is frequently assumed to be zero, as is the case here, which then presupposes that the planet absorbs all incident radiation and behaves as a blackbody. With the possible exception of cases where internal and atmospheric heating contributes to this temperature, the calculated temperature is likely to be overestimated.

Finally, many of the short-period planet discoveries (particularly those discovered using the transit method) assume a circular orbit and do not provide measurements of the eccentricity of argument of periastron. In those cases, we assume $e=0.0$ and $\omega=90^{\circ}$, which is equivalent to fixing the time of periastron passage to the predicted time of midtransit.

\subsection{Fundamental Calculations}

For each of the planets that meet the minimum parameter requirements described previously, we perform the following calculations. The first step is to calculate the extent of the
$\mathrm{HZ}$ based upon the properties of the host star. In order to do this, we require the luminosity of the host star, which is approximated as

$$
L_{\star}=4 \pi R_{\star}^{2} \sigma T_{\mathrm{eff}}^{4},
$$

where $\sigma$ is the Stefan-Boltzmann constant.

Using the boundary conditions of runaway greenhouse and maximum greenhouse effects at the inner and outer edges of the HZ, respectively (Underwood et al. 2003), the stellar fluxes at these boundaries are given by

$$
\begin{gathered}
S_{\text {inner }}=4.190 \times 10^{-8} T_{\text {eff }}^{2}-2.139 \times 10^{-4} T_{\text {eff }}+1.268 \\
S_{\text {outer }}=6.190 \times 10^{-9} T_{\text {eff }}^{2}-1.319 \times 10^{-5} T_{\text {eff }}+0.2341 .
\end{gathered}
$$

The inner and outer edges of the HZ are then derived from the following:

$$
r_{\text {inner }}=\sqrt{L_{\star} / S_{\text {inner }}} \quad r_{\text {outer }}=\sqrt{L_{\star} / S_{\text {outer }}},
$$

where the radii are in units of $\mathrm{AU}$ and the stellar luminosities are in solar units.

The next calculation performed is that of the planetary equilibrium effective temperature. Without any direct knowledge of the surface conditions or atmosphere of the planet, this calculation requires numerous assumptions that can be used to estimate a range of temperature values. One such assumption is that of the heat redistribution efficiency of the atmosphere, which depends upon zonal wind speeds. If the atmosphere is $100 \%$ efficient at redistributing heat ("well-mixed" model), the planetary equilibrium effective temperature is given by

$$
T_{p}=\left(\frac{L_{\star}(1-A)}{16 \pi \sigma r^{2}}\right)^{\frac{1}{4}}
$$

where $A$ is the spherical (Bond) albedo and $r$ is the star-planet separation. If the atmosphere is completely inefficient with respect to heat redistribution, then the effective temperature is

$$
T_{p}=\left(\frac{L_{\star}(1-A)}{8 \pi \sigma r^{2}}\right)^{\frac{1}{4}}
$$

which results in a hot dayside for the planet. The HZG provides the calculated values for both the periastron and apastron locations of the orbit. In the case of the movies (see $\S 4.3$ ), the wellmixed temperatures are calculated at each location of the orbit where a Keplerian solution is evaluated.

The final calculation is that of the Keplerian orbit. The true anomaly, $f$, is the angle between the position at periastron and the current position in the orbit measured at the focus of the ellipse. The mean anomaly is defined as 


$$
M=\frac{2 \pi}{P}\left(t-t_{p}\right)
$$

and it is hence the fraction of the orbital period that has elapsed since the last passage at periastron, $t_{p}$. From the mean anomaly we can calculate the eccentric anomaly, $E$, which is the angle between the position at periastron and the current position in the orbit, projected onto the ellipse's circumscribing circle perpendicularly to the major axis, measured at the center of the ellipse. These two quantities are related via Kepler's equation:

$$
M=E-e \sin E,
$$

which we solve via the Newton-Raphson method, and it has the solution

$$
E=\frac{M-e(E \cos E-\sin E)}{1-e \cos E} .
$$

This yields the value of $E$ and, hence, the value of $f$ :

$$
\cos f=\frac{\cos E-e}{1-e \cos E}
$$

The star-planet separation for eccentric planets has the following form:

$$
r=\frac{a\left(1-e^{2}\right)}{1+e \cos f} .
$$

Thus, we deduce from equations (3) and (4) that the eccentricity of a planetary orbit introduces a time dependency to the effective temperature of the planet.

\subsection{A Note on "Habitability"}

The question arises as to whether those planets that are found to lie within their host stars HZ are indeed habitable. This is unknown, since there are a variety of planetary properties that contribute toward habitability, in addition to the flux received from the host star (Schulze-Makuch et al. 2011). For example, many of the planets described here are giant planets that may not even have a rocky surface. Moons of those systems may have conditions more suitable to sustaining life, however (Kane \& Gelino 2010). The view of what makes a planet habitable has an inevitable anthropic selection effect that contributes to our selection of preferred targets (Waltham 2011). The survival of extremophiles in orbits that are not consistently within the $\mathrm{HZ}$ are also poorly understood, since we have no precedents for those conditions within our own system (Kane \& Gelino 2012). The variety of factors that thus influence the sustainability of life must be accounted for when considering if a planet within the $\mathrm{HZ}$ is truly a candidate for potentially hosting life.

\section{ONLINE SITE STRUCTURE}

Here, we describe the overall structure and design of the World Wide Web site and how updates are carried out.

\subsection{Data Extraction and Sorting}

The HZG is currently not a standalone service, but relies upon the data curation of the EDE. The HZG code is executed periodically to ensure that the catalog and data products remain in sync with the services of the EDE. When executed, the code uses a wget command to extract all of the current data from the EDE and to then identify the columns of the output file that are needed by the HZG. These columns are then sorted by the planet name and output to a new file that is ready to be processed by the site construction code.

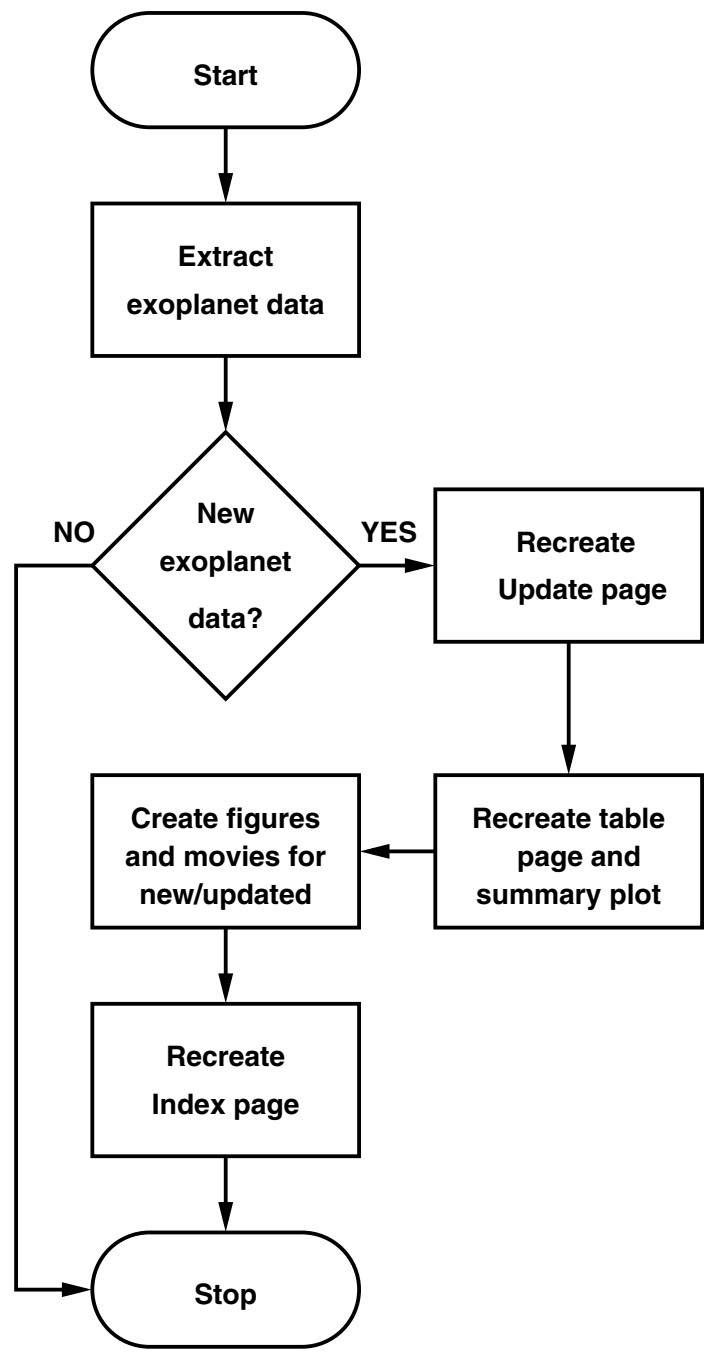

FIG. 2.-A flowchart that summarizes the automated reconstruction process wherever the HZG update code is executed. 
TABLE 2

Some Planets That Spend 100\% of Their TIME WITHIN HZ

\begin{tabular}{|c|c|c|c|c|}
\hline Planet & $\begin{array}{c}T_{\text {eff }}^{a} \\
(\mathrm{~K})\end{array}$ & $\begin{array}{l}T_{\text {eff }}^{b} \\
(\mathrm{~K})\end{array}$ & $\begin{array}{l}T_{\text {eff }}^{c} \\
(\mathrm{~K})\end{array}$ & $\begin{array}{l}T_{\text {eff }}^{d} \\
(\mathrm{~K})\end{array}$ \\
\hline tau Gru b & 279 & 235 & 260 & 219 \\
\hline mu Ara b & 322 & 271 & 283 & 238 \\
\hline Kepler-22b & 340 & 286 & 340 & 286 \\
\hline HD 99109b & 296 & 249 & 271 & 228 \\
\hline HD $45364 c$ & 341 & 286 & 309 & 260 \\
\hline HD 38801b & 349 & 294 & 349 & 294 \\
\hline HD $28185 b$ & 326 & 274 & 310 & 261 \\
\hline HD 23079b & 302 & 254 & 272 & 229 \\
\hline HD $221287 b$ & 358 & 301 & 330 & 278 \\
\hline HD $188015 b$ & 328 & 276 & 286 & 240 \\
\hline HD 16760b & 288 & 243 & 270 & 227 \\
\hline HD $108874 b \quad$. & 348 & 293 & 306 & 257 \\
\hline HD 10697b & 289 & 243 & 262 & 220 \\
\hline HD $10180 \mathrm{~g}$ & 296 & 249 & 296 & 249 \\
\hline $55 \mathrm{Cnc} \mathrm{f}$ & 328 & 276 & 328 & 276 \\
\hline
\end{tabular}

NoTE. $-T_{\text {eff }}^{a}$ : periastron, hot dayside; $T_{\text {eff }}^{b}$ : periastron, wellmixed; $T_{\text {eff }}^{c}$ : apastron, hot dayside; $T_{\text {eff }}^{d}:$ apastron, well-mixed.

The HZG retains a copy of the previous data file for the purposes of comparison. Specifically, the code checks for new planets or any changes that may have occurred in the parameters for known planets. If no changes are detected, then the code halts at this point, and any alterations that may have been made are restored.

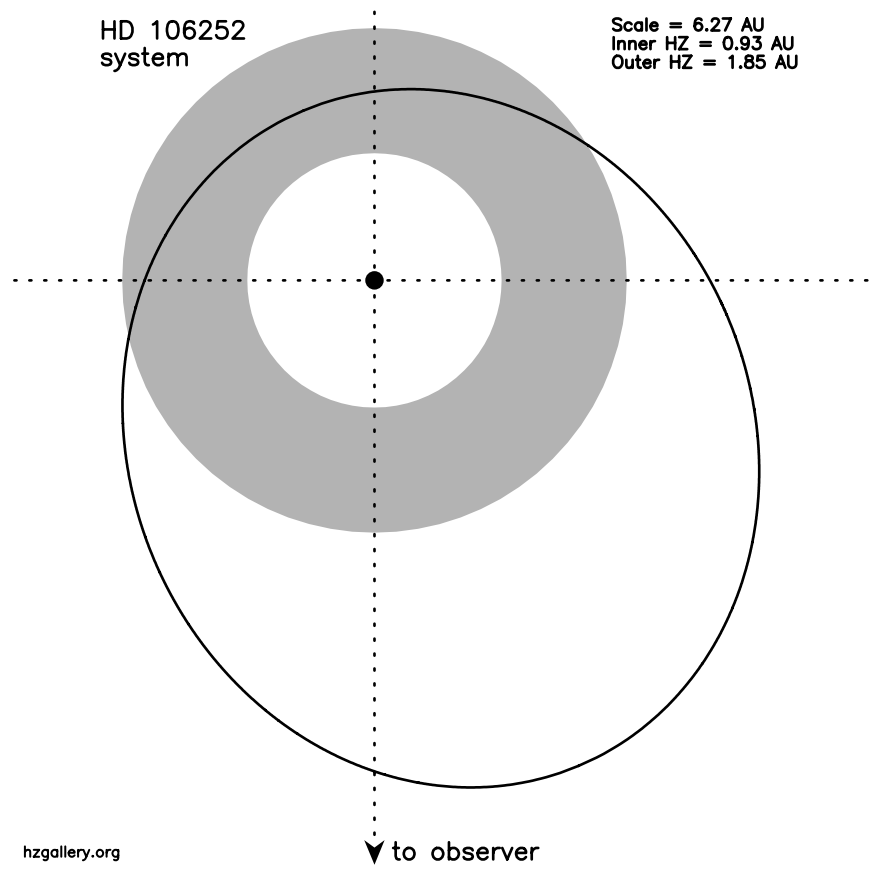

\subsection{Automated Reconstruction}

The philosophy of the HZG design is accuracy and sustainability such that it can continue to provide useful results that keep pace with the current rate of exoplanet detection. The optimal path to reach this goal is to avoid data entry, which dramatically increases maintenance and the introduction of errors. The site update is initiated via a single command that executes a shell script. As described in $\S 4.1$, the script first retrieves the most recent data and checks for changes. If there are new planets and/or parameters, then the script proceeds to execute a series of FORTRAN programs that perform the necessary calculations and HTML modifications to incorporate the additional information.

The flowchart shown in Figure 2 summarizes how the update and reconstruction of the Web site functions. When changes to the database are necessary, the update page is modified with new planets added and/or changes to planetary parameters. The next step is to update the summary plot shown on the main page and the table page. It is computationally inexpensive to completely recreate these items, rather than to attempt to only modify those values that have changed. However, the next step of creating the figures in the gallery and the movies is timeconsuming, and so this step is only initiated where changes are required. The script finally recreates the index (main) page before stopping. The script also has an optional flag that, if used when executed, forces the complete reconstruction of the entire site, including all the figures and movies.

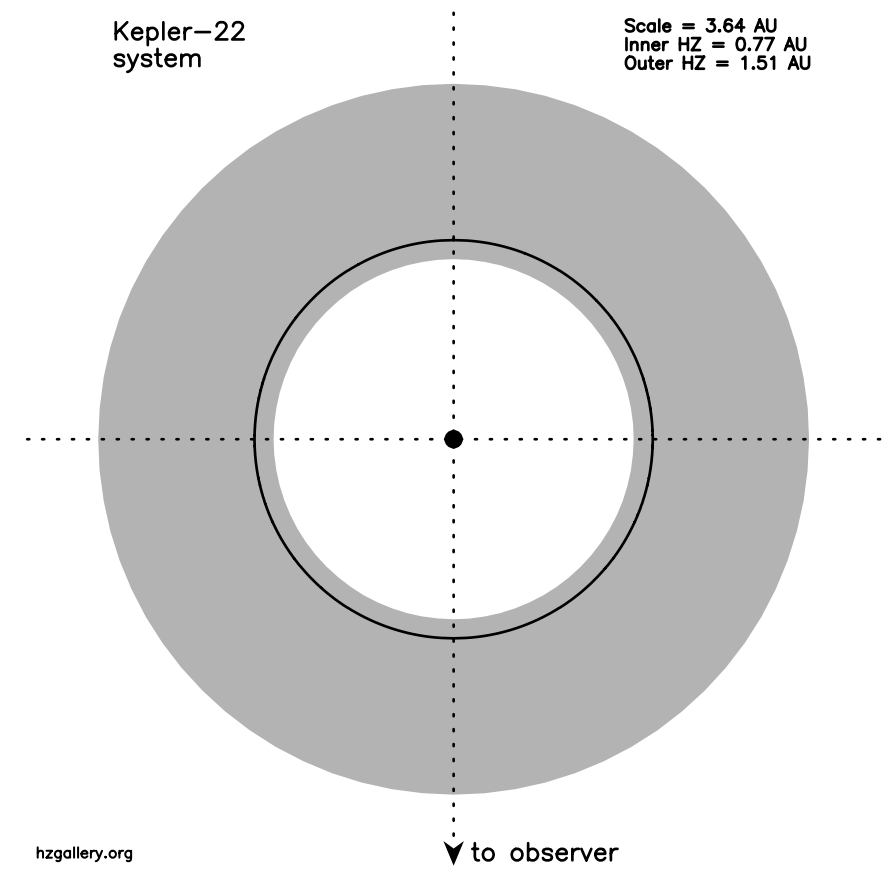

FIG. 3.-Two examples of orbits represented in the gallery section of the HZG: HD 106252b (left) and Kepler-22b (right) 
The revised version of the site is built at a temporary location where the contents may be reviewed for accuracy and bug-related issues. Once satisfied that the update has been successfully completed, the new version is transferred to the operational location.

\subsection{The Table, Gallery, and Movies}

The three main services provided by the HZG are a table of derived planetary properties, a gallery of the planetary orbits with respect the their HZ, and movies that provide a visual aid for the varying predicted planetary temperatures.

The table includes the basic orbital properties of the planet, the percentage of the orbital phase spent within the HZ, and calculated temperatures at apastron and periastron for both the well-mixed and hot-dayside models (see § 3.3). The table is sortable on any of these columns such that it can used to easily select targets of interest. For example, shown in Table 2 is a subset of the complete table in the HZG, which includes those planets that spend $100 \%$ of their time within their stars HZ. We have only included the temperature columns in this truncated table. The HZG additionally allows the user to click on any of the planet names to load the location of that system with the gallery section.

The gallery section includes up to three plots per system, depending in the location of inner and outer planets with respect to their HZ. The purpose of this is to accommodate the plotting of systems whose planetary orbits occupy a wide range of orbital radii. In Figure 3 are shown two examples from the HZG that demonstrate the diversity of these figures. HD $106252 \mathrm{~b}$ is in a 1531 day orbit whose periastron occurs within the HZ of its star. Only $17 \%$ of the total orbital phase occupies the HZ though, since the planet is moving faster near periastron. This may be contrasted with the case of Kepler-22b, also shown in Figure 3. The discovery of this planet was recently announced by Borucki et al. (2012). Independent calculations by the HZG code confirm that the planet does indeed spend $100 \%$ of the orbit within the HZ.

Finally, the movies section of the HZG provides users with animations for individual planetary orbits, available in MPEG-2 and MPEG-4 formats. The animations track the star-planet separation and calculated effective temperatures for the wellmixed atmospheric model. This is particularly useful for eccentric orbits, where the temperature range can be substantial. The animations are divided into 200 frames of equal time increments per orbit, which aids in the animation resolution for highly eccentric orbits during periastron passage.

\section{CONCLUSIONS}

The rate of exoplanet discoveries is continuing to increase, both with the radial velocity and transit methods. As the sensitivity and time baseline of the methods improve, the orbits of the discovered planets are increasingly being found to lie in the HZ of their host stars or beyond. This is a crucial step in identifying planets that are best suited for follow-up activities related to habitability and astrobiology.

The HZG seeks to provide a service in an easily maintainable way in order to keep up with the discovery rate. The HZG mostly includes planets whose host stars are relatively bright, since they were primarily discovered using the radial velocity technique. The advantage of this is that these stars lend themselves to more accessible follow-up observations that can be better used to characterize those planets.

The structure of the site allows for automatic updates and reconstruction in a very short period of time. The figures and movies are intended for use in both scientific and public contexts and can be easily transported into any presentation. The tools allow the user to determine which known planets spend substantial time in their habitable zones and to visualize their orbits and also perform general investigations into the demographics of these targets. The HZG will continue to adapt to the needs of the exoplanet community and further develop tools as needed.

The authors would like to thank Ravikumar Kopparapu, Lisa Kaltenegger, and Jason Wright for their useful feedback on improvements to the Habitable Zone Gallery. This research has made use of the Exoplanet Orbit Database and the Exoplanet Data Explorer at exoplanets.org.

\section{REFERENCES}

Baraffe, I., Chabrier, G., Barman, T. S., Allard, F., \& Hauschildt, P. H. 2003, A\&A, 402, 701

$\rightarrow$ Borucki, W. J., et al. 2011a, ApJ, 728, 117

$\rightarrow \longrightarrow$. 2011b, ApJ, 736, 19

$\rightarrow-$. 2012, ApJ, 745, 120

$\rightarrow$ Fressin, F., et al. 2012, Nature, 482, 195

Gautier, T. N., et al. 2012, ApJ, preprint (arXiv:1112.4514)

$\rightarrow$ Jones, B. W., \& Sleep, P. N. 2010, MNRAS, 407, 1259

$\rightarrow$ Kaltenegger, L. 2010, ApJ, 712, L 125

$\rightarrow$ Kaltenegger, L., \& Sasselov, D. 2011, ApJ, 736, L25

$\rightarrow$ Kane, S. R., \& Gelino, D. M. 2010, ApJ, 724, 818

2011, ApJ, 741, 52

2012, Astrobiology, submitted $\rightarrow$ Kasting, J. F., Whitmire, D. P., \& Reynolds, R. T. 1993, Icarus, 101, 108

$\rightarrow$ Kipping, D. M., Fossey, S. J., \& Campanella, G. 2009, MNRAS, 400, 398

$\rightarrow$ Schulze-Makuch, D. 2011, Astrobiology, 11, 1041

$\rightarrow$ Selsis, F., Kasting, J. F., Levrard, B., Paillet, J., Ribas, I., \& Delfosse, X. 2007, A\&A, 476, 1373

Smalley, B. 2005, Mem. Soc. Astron. Ital. Suppl., 8, 130

$\rightarrow$ Swift, D. C., et al. 2012, ApJ, 744, 59

$\rightarrow$ Underwood, D. R., Jones, B. W., \& Sleep, P. N. 2003, Int. J. Astrobiology, 2, 289

$\rightarrow$ Waltham, D. 2011, Icarus, 215, 518

$\rightarrow$ Wright, J. T., et al. 2011, PASP, 123, 412 